\title{
Article
}

\section{The impact of a night confinement policy on patients in a high secure inpatient mental health service.}

Chu, Simon, McNeill, Kimberley, Wright, Karen Margaret, Hague, Anthony and Wilkins, Tracy

Available at http://clok.uclan.ac.uk/12902/

Chu, Simon ORCID: 0000-0001-8921-4942, McNeill, Kimberley, Wright, Karen Margaret ORCID: 0000-0003-0693-7294, Hague, Anthony and Wilkins, Tracy (2015) The impact of a night confinement policy on patients in a high secure inpatient mental health service. Journal of Forensic Practice, 17 (1). pp. 21-30. ISSN 2050-8794

It is advisable to refer to the publisher's version if you intend to cite from the work. dx.doi.org/10.1108/JFP-11-2014-0045

For more information about UCLan's research in this area go to

http://www.uclan.ac.uk/researchgroups/ and search for <name of research Group>.

For information about Research generally at UCLan please go to http://www.uclan.ac.uk/research/

All outputs in CLoK are protected by Intellectual Property Rights law, including Copyright law. Copyright, IPR and Moral Rights for the works on this site are retained by the individual authors and/or other copyright owners. Terms and conditions for use of this material are defined in the policies page. 
Simon Chu, Kimberley McNeill, Karen M. Wright, Anthony Hague, Tracy Wilkins, (2015). The impact of a night confinement policy on patients in a UK high secure inpatient mental health service. Journal of Forensic Practice, 17(1), $21-30$.

DOI: http://dx.doi.org/10.1108/JFP-11-2014-0045

The impact of a night confinement policy on patients in a high secure inpatient mental health service.

Simon Chu, Kimberley McNeill, Karen Wright, Anthony Hague, Tracy Wilkins

\section{Abstract}

Purpose - From 2012, all high-secure forensic mental health services in England began operating a policy of confining patients to their locked bedrooms overnight to increase service efficiency and reduce costs. The purpose of this paper is to assess the views of staff and patients concerning the policy and examine the specific impact of the policy on patients.

Design/methodology/approach - Measures of patients' sleep hygiene, patients' behaviour, ward atmosphere, engagement with therapy and adverse incidents were taken both before and after the night confinement (NC) policy was implemented. Both patients and staff also expressed their views of the impact of the NC policy.

Findings - Results provide converging evidence that the impact of the NC policy on patients is negligible. There were no consistent negative effects of confining patients overnight. Rather, patients and staff were broadly positive about the impact that the practice had on patients.

Practical implications - Confining patients to locked bedrooms overnight does not exert any consistent influence, positive or negative, on patients' sleep hygiene, behaviour or engagement with therapy, and patients expressed a broadly positive view of the practice of NC. Thus, a NC policy may have a contribution to make to the provision an effective high-secure mental health service.

Originality/value - The study provides convincing evidence that secure inpatient mental health services that are considering the adoption of a NC policy may do so without fear of a negative impact on patients.

Keywords Forensic mental health, Night confinement, Secure service, Sleep, Therapy engagement, Ward atmosphere

\section{Introduction}

Until 1995, it was standard practice in high security hospitals in England to confine patients to their locked rooms at night. In response to the report that emerged from the Blom-Cooper Inquiry (HMSO, 1992), this practice was ended in 1995 at which point all high security hospitals in England began providing around-the-clock nursing care. More recently however, the NHS has come under increasing financial strain with growing pressure to reduce costs and increase operating efficiency. In 2011, the directions from the Secretary of State for Health concerning safety and security at the high secure hospitals in England were again revised to allow for the locked confinement of patients to their rooms overnight, a policy referred to here as night confinement (NC). Since then, all three high security psychiatric hospitals have implemented a policy of NC.

The central motivation for the commencement of $\mathrm{NC}$ has been to achieve a reduction in costs of nighttime ward staff, and while the potential financial benefits are considerable, there was clearly a need 
to assess the effects of the policy on the patient population in the hospital. At the outset, confining patients to their rooms may appear to be an unwanted and unwarranted imposition with the implication that, for patients, the policy would be detrimental and unpopular. However, the true effects of such a policy of confinement are not yet clearly understood; while some outcomes of confinement may be negative, it is possible that some outcomes may be positive. When Rampton Hospital evaluated the impact of the implementing their NC policy across the hospital in 2011, they found that the measurable impact of NC was quite small (Braham, Heasley \& Akiens, 2013). Their evaluation included assessments of ward climate and patient quality of life, as well as staff working environment and an analysis of adverse incident and seclusion rates. Interviews were also conducted with patients and staff and the consistent finding across all the data was that there were no adverse effects of implementing the NC policy. Nevertheless, a number of practical and methodological issues involved in that evaluation led the researchers to conclude that further evaluation was necessary if $\mathrm{NC}$ was to be adopted more widely across the high secure estate.

The present programme of research aimed to clarify the effect that NC may potentially have had on patients in the hospital and broaden the investigation to include wider issues that may have been affected by the implementation of the NC policy. Specifically, as well as surveying the patient (and ward staff) perceptions of the impact of NC, the present project examined the effect of NC on overall ward climate as well as patients' gross behaviour, engagement with therapy, sleep quality, and adverse incident rates.

Anecdotal reports suggest that, before the implementation of the night confinement policy, the majority of patients tended to retire to bed late and wake late, or woke during the night and took naps during the daytime. Thus the external imposition of structure to the night may have the effect of altering (and perhaps improving) sleep hygiene (the amount and the quality of sleep achieved) such that patients' sleep patterns may have become more regular, thus resulting in better quality sleep. Alternatively, it is also possible that externally imposed structure may have a negligible effect on sleep patterns. One aim of the present project therefore was to assess through patients' self-report the general amount and quality of patients' sleep both before and after NC. If an improvement in sleep hygiene a result of NC is a possibility, the psychological benefits may be considerable. For example, daytime wakefulness, mood, attention and motivation may improve because these aspects of human behaviour are all known to be adversely affected by poor sleep hygiene (McCoy \& Strecker, 2011). Therefore, one aspect of the present study assessed gross behaviour and interpersonal style using observations and assessments from ward staff to see if NC impacted in any significant way.

If positive changes in sleep hygiene affect mood and interaction in a positive manner, one might reasonably expect that ward atmosphere may change for the better and so a further aim of the study indexed ward atmosphere before and after NC was implemented to investigate whether NC exerted any effect on social climate on the ward. Recent evidence also suggests that ward atmosphere is a strong determinant of engagement with therapy. According to Beazley and Gudjonsson (2011), a positive social climate on a ward may have positive effects on patients' engagement with treatment, and for patients, the ultimate outcome of improvements in mood, attention and motivation may be an improvement in the degree to which they engage with therapy. Therapy engagement has been a somewhat amorphous term that has been conflated with the concepts of treatment motivation, working alliance and treatment progression amongst others (Tetley et al., 2011). Therapy engagement is defined here as the extent to which the client actively participates in the treatment on offer, and encompasses physical attendance at therapy sessions and a willingness to share thoughts, feelings, problems and history (Krause, 1967). It also involves a willingness to develop effective working relationships with therapist and others in treatment (Siebel \& Dowd, 1999). A further aspect of the present study was therefore to examine prospective changes in therapy engagement as a potential consequence of NC. 
In order to assess the possible changes in overall safety and security, we also examined adverse incident records prior to and subsequent to the commencement of NC. Finally, we also assessed patient and ward staff views on the impact of the NC policy on patients. In sum, our aim was to garner a comprehensive view of the impact of the night confinement policy on patients.

\section{Method}

\section{Overview}

The night confinement policy was implemented at Ashworth Hospital gradually over a 14-month period, beginning with two wards in October 2012 and finishing with the final two wards in November 2013. Under the NC policy at Ashworth, patients are confined to their rooms at 21:15hrs until 07:15hrs the following morning. During the period of confinement, patients are directly observed every 30 minutes. The present study examined the impact of the NC policy on the final six wards to implement NC between September and November 2013. We approached patients to complete a set of measures 4-6 weeks before NC was due to commence on their ward and returned to collect responses again approximately 12 weeks after NC began on their ward. We also gathered pre- and post-NC data from ward nursing staff at these times and also data from therapists who were working with patients on participating wards. In all cases, pre-NC data was compared to post-NC data in order to assess the impact of NC on those factors.

\section{Measures}

Staff and patient attitudes towards night confinement and the impact of confinement was assessed here using a bespoke 21-item survey examining opinions about different aspects of NC. The survey addresses 11 issues that emerged from brief surveys of patient's views after NC was introduced at Rampton Hospital and Ashworth Hospital. Issues included views of potential changes in patient mood, patient sleep, amount of support available to patients at night, changes in patients' mental health, patient safety, etc. Each issues was addressed with two items (one item reverse scored) apart from the issue concerning the overall attitude towards night confinement which was addressed with one item.

Ward climate was measured using the Essen Climate Evaluation Schema (EssenCES; Schalast et al., 2008), a standard 17-item measure of ward atmosphere with three subscales measuring patient cohesion, perception/experience of safety, and therapeutic hold, that has been validated in secure settings (Howells et al., 2008; Tonkin et al., 2012). Both patients and staff on participating wards completed the EssenCES.

Patients' sleep hygiene was measured using the Pittsburgh Sleep Quality Index (PSQI; Buysse et al., 1988), a 9-item self-report measure of the sleep quality that the participant has experienced over the previous month, measuring sleep latency, duration, efficiency, and a range of other indices of sleep quality.

Patient behaviour was indexed in three different ways. Gross behaviour was assessed through ward staff assessments of each patient using the CIRCLE nurses' observation scale (Blackburn \& Renwick, 1996) which measures the interpersonal style and quality of interaction exhibited by a patient in a closed living environment. It is a 51-item measure completed by ward staff about individual patients with whom they are very well acquainted and indexes 8 characteristics of patient behaviour and interaction: dominant, coercive, hostile, withdrawn, submissive, compliant, nurturant and gregarious. Higher scores for a characteristic indicate higher levels of that characteristic. 
If patients on participating wards were engaged in one-to-one therapy at the time of pre-NC data collection, their therapists were asked to complete the Hall therapy engagement measure (Hall et al., 2001), a 10-item measure of a patient's level of engagement in therapy, assessing communication, collaboration and compliance with therapy and the therapeutic process. This was completed for all patients from the participating wards who were engaged in individual therapy but was not limited to the therapists of patients who were themselves participating. At the time of post-NC data collection, therapists who were still engaged in therapy with those patients were asked to complete the measure a second time.

The third means of assessing patient behaviour was through the routinely collected adverse incident records. For each participating ward, we collated the number of adverse incidents reported for the 24-week period immediately preceding the commencement of NC and for the 24-week period immediately following NC.

Procedure and sampling

For each patient on participating wards, we approached the Responsible Clinician (RC) for each patient, described the nature of the study and the requirements, and asked for permission to approach each patient for participation in the study. Once RC permission to approach was granted, a research assistant approached patients on wards and explained the nature and purpose of the study. Patients were then free to volunteer their participation or to decline. 4-6 weeks before NC was due to begin on a participating patient's ward, patients completed measures of sleep quality, ward atmosphere, and their attitudes towards NC. Ward staff were asked to complete the CIRCLE nurses observation scale for patient on the participating wards, the EssenCES scale the survey of attitudes towards NC. In addition, therapists were asked to complete the therapy engagement measure for each of their patients from the relevant wards. In all cases, the same respondents were asked to complete the same measures again $12-14$ weeks after NC began.

There were 99 patients on the six participating wards. RCs granted permission to approach 84 patients and 41 of these agreed to participate. Before the completion of the post-confinement data collection phase, 11 patients withdrew from the study either because they no longer wished to participate or because they had left the hospital. 30 patients (mean age: 39 , range $23-60$ years) completed the study in full. 14 patients had a main diagnosis of schizophrenia, 10 patients were solely diagnosed as having a personality disorder while the remaining 6 patients were diagnosed with a combination of mental illness and personality disorder.

Staff contacted to participate in the research as and when their input was required. Questionnaire packs were sent to wards for the attention of ward staff. 144 ward staff returned completed pre- and post-NC responses for the attitudes survey and 119 ward staff returned completed pre- and post NC responses for the EssenCES.

Ethical approval for this research was granted by the Health Research Authority National Research Ethics Committee (REC reference: 13/NW/0391).

\section{Results}

Sleep quality

Scores on the Pittsburgh Sleep Quality Index (PSQI) index sleep disturbance and thus higher scores indicate greater levels of disturbed sleep. The published norm for the PSQI in the general non-clinical 
Table 1. Mean CIRCLE observation scale scores for each of 8 dimensions pre-confinement and post-confinement, and the $\mathrm{p}$-values resulting from paired-samples t-test comparisons.

\begin{tabular}{lcccc} 
subscale & pre & post & $t$ & $p$ \\
\hline dominant & -0.71 & -0.66 & 0.55 & 0.584 \\
coercive & -0.40 & -0.47 & 1.02 & 0.309 \\
hostile & -0.29 & -0.05 & 1.93 & 0.017 \\
withdrawn & -0.11 & -0.30 & 2.44 & 0.057 \\
submissive & 0.44 & 0.49 & 0.47 & 0.641 \\
compliant & 0.29 & 0.35 & 0.47 & 0.637 \\
nurturant & 0.34 & 0.33 & 0.98 & 0.921 \\
gregarious & 0.47 & 0.60 & 0.10 & 0.327 \\
\hline
\end{tabular}

Note: Scores are in the form of z-scores, with higher scores indicating higher levels of a particular characteristic. The $\alpha$ level for the analyses was adjusted to 0.00625 to allow for multiple comparisons, $d f=85$.

population is 2.7 (SD: 1.7) and for a depressed sample is 11.1 (SD: 4.3; Buysse et al., 1988). The mean pre-NC PSQI score was 6.9 (SD: 3.9) and the mean post-NC score was 5.8 (SD: 3.6). A two-tailed paired samples t-test indicated that the reduction in PSQI scores was not statistically significant, $t(29)=1.57$, $p=0.127$.

Change scores were variable but importantly, confinement was not associated with any consistent increase in sleep disturbance. Of the 30 participating patients, 11 patients reported greater sleep disturbance than before, 3 reported no change, and 16 reported an improvement in sleep quality.

\section{Gross behaviour}

Ward staff provided complete pre- and post-NC ratings for 86 patients and for $41 \%$ of patients, ratings at both time points were provided by the same nurse. Mean scores for each of the eight dimensions in both their pre- and post-NC phase of the data are shown in Table 1 along with the results of pairedsamples t-test comparisons in each dimension. Positive changes indicate an increase in the observed frequency of that type of behaviour. After adjusting for multiple comparisons ( $\alpha=.0062$ ), analyses showed no significant changes in any of the dimensions and thus the staff ratings of patients' general behaviour indicated that there were no consistent changes to patient behaviour between the two time points.

\section{Ward atmosphere}

30 patients and 119 ward staff completed the EssenCES at pre- and post-confinement. Table 2 shows the scores for each of the three subscales on the EssenCES from patient and staff assessments of the ward climate before and after NC began, as well as the result of paired samples t-tests comparing preand post-NC scores on each subscale for each group. The scores for ward climate are at least comparable to published norms for a high secure sample (based on data from Rampton Hospital; Howells et al., 2009). Comparing pre- and post-NC scores on each subscale suggested that in the assessment of both patients and staff there were no significant changes in ward climate between the two time points.

Engagement with therapy

Therapists completed the Hall Therapy Engagement Measure for any patients with whom they were working both before and after NC commenced. 28 patients met the criteria. On the Hall Therapy 
Table 2. Mean EssenCES scores (standard deviation) from patients and staff for each of the three subscales for pre- and post-confinement and $p$-values from paired-samples t-tests to compare preand post-NC, as well as the norms for each subscale for secure settings.

\begin{tabular}{|c|c|c|c|c|c|c|c|c|}
\hline \multirow[b]{2}{*}{ subscale } & \multicolumn{4}{|c|}{ patient } & \multicolumn{4}{|c|}{ staff } \\
\hline & pre-NC & post-NC & $t$ & $p$ & pre-NC & post-NC & $t$ & $p$ \\
\hline patient cohesion & $\begin{array}{c}9.4 \\
(5.8)\end{array}$ & $\begin{array}{l}10.7 \\
(5.7)\end{array}$ & 1.31 & 0.201 & $\begin{array}{c}8.5 \\
(4.2)\end{array}$ & $\begin{array}{c}8.3 \\
(4.3)\end{array}$ & 0.55 & 0.309 \\
\hline experienced safety & $\begin{array}{l}11.9 \\
(5.3)\end{array}$ & $\begin{array}{l}12.0 \\
(5.7)\end{array}$ & 0.21 & 0.833 & $\begin{array}{l}10.8 \\
(4.2)\end{array}$ & $\begin{array}{l}11.0 \\
(4.8)\end{array}$ & 1.61 & 0.685 \\
\hline therapeutic hold & $\begin{array}{l}14.0 \\
(3.8)\end{array}$ & $\begin{array}{l}12.4 \\
(5.0)\end{array}$ & 2.07 & 0.048 & $\begin{array}{l}16.2 \\
(2.9)\end{array}$ & $\begin{array}{l}16.3 \\
(2.6)\end{array}$ & 0.07 & 0.945 \\
\hline
\end{tabular}

Note: higher scores indicate higher levels of patient cohesion, safety and therapeutic hold. The a level for all analyses was adjusted to 0.016 to allow for multiple comparisons, $d f=27$.

engagement measure, good engagement is indicated by scores above a cut-off of 33 . In our patient sample, 26 out of 28 patients met the cut-off before NC commenced, and this increased to 27 out of 28 patients post-NC. The mean pre-NC score was 40.3 (SD: 5.3 ) and the mean post-NC score was 41.5 (SD: 5.5). A paired samples t-test showed that there was no statistical difference in the pre- and postNC engagement scores for our sample, $t(27)=1.43, p=0.16$. This indicates that no deterioration in engagement with therapy was associated with the implementation of night confinement and if anything, there were indications that patients engaged a little better with therapy post-NC.

\section{Adverse incidents}

We extracted the absolute number of adverse incidents recorded on each of the six participating wards for the 24-week period leading up to the commencement of NC on that ward and the 24-week period immediately following. These are shown in Table 3 where it can be seen that for the vast majority of wards, there are no appreciable differences between pre- and post-NC incident rates apart from on ward $B$ where there is a notable increase in incidents. The substantial increase in the total number of incidents in the whole sample, from 193 pre-NC to 282 post-NC, may largely be traced back to the increase on ward B. Indeed, without the inclusion of ward B, there is no real difference in total incidents between pre- and post-confinement. Anecdotal evidence from ward B suggests that there were specific reasons (i.e. particular changes in patient population) for the increase in adverse incidents on that ward during this period that were unrelated to the implementation of night confinement. Furthermore, ward B was the only ward to show such a change in incident rates over this period, suggesting that the increase in incident rates was not likely to be related to the

Table 3. Total number of adverse incidents reported in the 24-week period leading up to confinement on each ward and the 24-week period immediately following confinement.

\begin{tabular}{ccc} 
ward & before NC started & after NC started \\
\hline A & 30 & 20 \\
B & 66 & 143 \\
C & 1 & 8 \\
D & 24 & 26 \\
E & 33 & 46 \\
F & 39 & 39 \\
\hline total & 193 & 282 \\
& & \\
total without B & 127 & 139 \\
\hline
\end{tabular}


Table 4. The proportion of patients whose attitudes were positive, negative and neutral concerning each of the issues in relation to night confinement before confinement started and afterwards.

\begin{tabular}{|c|c|c|c|c|c|c|}
\hline \multirow[b]{2}{*}{ Possible change } & \multicolumn{3}{|c|}{ before NC started } & \multicolumn{3}{|c|}{ after NC started } \\
\hline & 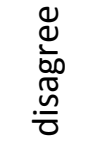 & 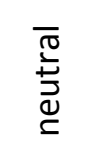 & $\underset{\substack{\Delta 0 \\
\sigma}}{\stackrel{\Xi}{d}}$ & 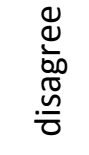 & 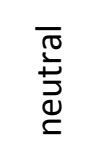 & 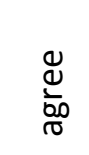 \\
\hline Changes were communicated well beforehand & - & - & - & $3 \%$ & $0 \%$ & $97 \%$ \\
\hline Social interaction between patients is better & $47 \%$ & $30 \%$ & $23 \%$ & $33 \%$ & $33 \%$ & $33 \%$ \\
\hline Interaction between patients and staff is better & $43 \%$ & $27 \%$ & $30 \%$ & $33 \%$ & $27 \%$ & $40 \%$ \\
\hline Patient mental health improves & $50 \%$ & $20 \%$ & $30 \%$ & $10 \%$ & $30 \%$ & $60 \%$ \\
\hline Support is available to patients during the night & $50 \%$ & $20 \%$ & $30 \%$ & $27 \%$ & $17 \%$ & $\mathbf{5 7 \%}$ \\
\hline Patient daytime safety is better & $40 \%$ & $30 \%$ & $30 \%$ & $23 \%$ & $40 \%$ & $37 \%$ \\
\hline Patient night safety is better & $37 \%$ & $23 \%$ & $40 \%$ & $30 \%$ & $30 \%$ & $40 \%$ \\
\hline Patient sleep is better & $33 \%$ & $10 \%$ & $57 \%$ & $20 \%$ & $17 \%$ & $63 \%$ \\
\hline Patient mood is better & $47 \%$ & $20 \%$ & $33 \%$ & $27 \%$ & $17 \%$ & $57 \%$ \\
\hline Incident rate is better & $30 \%$ & $23 \%$ & $47 \%$ & $17 \%$ & $30 \%$ & $53 \%$ \\
\hline Patients feel ok about being confined at night & $60 \%$ & $20 \%$ & $20 \%$ & $27 \%$ & $7 \%$ & $63 \%$ \\
\hline Overall attitude towards night time confinement & $47 \%$ & $20 \%$ & $33 \%$ & $23 \%$ & $30 \%$ & $47 \%$ \\
\hline
\end{tabular}

Note: The emboldened figures indicate the most popular response type for that item.

implementation of NC. Nevertheless, a permutations test for paired replicates indicated that the there was no difference in the level of adverse incidents on the six participating wards between the two time points, $p=0.141$.

Patient's attitudes about the impact of night time confinement

30 patients completed the attitudes survey both before and after NC. The survey asked for attitudes towards 23 issues related to confinement over the night time period and the proportion of patients who agreed, disagreed or expressed a neutral view at the two time points are presented in Table 4. Most striking from these data is the change in the pattern of responses between the two time points, from a largely negative attitude pre-NC, to a largely positive attitude post-NC. Before NC commenced, respondents believed that many issues (such as patients' mental health and the amount of support available at night) would deteriorate. $60 \%$ of patients had negative feelings about being confined at night and overall, $47 \%$ of patients expressed a negative attitude towards the policy. However several months after night confinement commenced, patients were more positive about the policy and their experiences of it. $60 \%$ of patients agreed that patients' mental health had improved, $57 \%$ agreed that support was available to patients at night, and $57 \%$ reported that patients' mood had improved since confinement started. With the exception of one issue (patient engagement with daytime activity), attitudes consistently shifted towards being more positive.

Staff attitudes about the impact of night time confinement on patients

The survey that was distributed to patients was also distributed to ward staff; therefore, the focus of the survey was again on what the potential impact of the policy would be on patients, rather than on the potential impact on staff. 144 ward staff completed the survey pre- and post-NC and a breakdown of responses is shown in Table 5.

Before NC was implemented, staff views of the impact of NC on patients was more positive than those of the patients themselves. In contrast to patients, staff thought that support would be available to 
Table 5. The proportion of staff whose attitudes were positive, negative and neutral concerning each of the issues in relation to night confinement before confinement started and afterwards.

\begin{tabular}{|c|c|c|c|c|c|c|}
\hline \multirow[b]{2}{*}{ Possible change } & \multicolumn{3}{|c|}{ before NC started } & \multicolumn{3}{|c|}{ after NC started } \\
\hline & 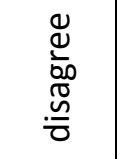 & 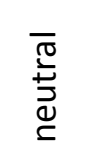 & 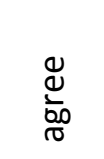 & 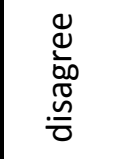 & 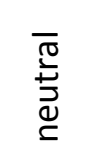 & 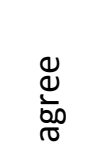 \\
\hline Social interaction between patients is better & $34 \%$ & $23 \%$ & $43 \%$ & $15 \%$ & $35 \%$ & $50 \%$ \\
\hline Interaction between patients and staff is better & $26 \%$ & $38 \%$ & $37 \%$ & $15 \%$ & $31 \%$ & $53 \%$ \\
\hline Patient mental health improves & $27 \%$ & $27 \%$ & $46 \%$ & $10 \%$ & $27 \%$ & $63 \%$ \\
\hline Support is available to patients during the night & $17 \%$ & $13 \%$ & $70 \%$ & $12 \%$ & $9 \%$ & $79 \%$ \\
\hline Patient daytime safety is better & $10 \%$ & $40 \%$ & $49 \%$ & $12 \%$ & $33 \%$ & $55 \%$ \\
\hline Patient night safety is better & $34 \%$ & $39 \%$ & $27 \%$ & $25 \%$ & $38 \%$ & $37 \%$ \\
\hline Patient sleep is better & $9 \%$ & $24 \%$ & $67 \%$ & $100 \%$ & $0 \%$ & $0 \%$ \\
\hline Patient mood is better & $36 \%$ & $26 \%$ & $38 \%$ & $18 \%$ & $32 \%$ & $50 \%$ \\
\hline Incident rate is better & $29 \%$ & $46 \%$ & $25 \%$ & $19 \%$ & $42 \%$ & $38 \%$ \\
\hline Patients feel ok about being confined at night & $48 \%$ & $27 \%$ & $25 \%$ & $37 \%$ & $24 \%$ & $39 \%$ \\
\hline Overall attitude towards night time confinement & $100 \%$ & $0 \%$ & $0 \%$ & $100 \%$ & $0 \%$ & $0 \%$ \\
\hline
\end{tabular}

Note: The emboldened figures indicate the most popular response type for that item.

patients at night, that patients would sleep better and their mental health would improve. Attitudes towards most issues became more positive after NC began as staff witnessed the impact of NC on patients. The proportion of negative attitudes towards different issues shrank except in the case of one or two notable exceptions. Previously, staff were mostly optimistic that patients would sleep better when night confinement started. However, post-confinement, they uniformly expressed the opinion that patient sleep had deteriorated. This is unusual given that there is little evidence from the patient responses that sleep had suffered in any consistent manner. Rather, it is likely that the staff responses were perhaps disproportionately affected by knowledge of one or two patients whose sleep hygiene had suffered. On a separate issue, a third of staff thought that daytime activity engagement was better than before, but the majority of staff disagreed. This chimed with the patient responses concerning engagement with daytime activities, indicating that there may still be some work to do in order to effectively engage patients in activities.

It is interesting that despite the largely positive view of staff concerning the impact of NC on patients, their overall attitude towards the NC policy is negative. Every member of staff, without exception, expressed a negative view overall both before and after NC was implemented. The fact that there was no change at all in attitudes from pre- to post-NC (when responses on all other items changed at least a little) implies that the staff response to this issue was irrespective of the impact of NC and was driven more by staff concerns about the policy that were quite independent of the impact on patients.

\section{Discussion}

The night confinement policy that has been implemented across the high secure hospital estate has been somewhat controversial in part because of concerns that the impact on patients would be negative. To consider these issues, we surveyed patient and staff views of the impact of the policy and assessed ward climate, sleep hygiene, gross behaviour, and therapy engagement both before and after implementing the night confinement policy on six wards in a high security mental health inpatient service. The results showed no significant changes, positive or negative, in any of the metrics that were used here and the attitudes of both patients and staff were broadly positive, suggesting that 
on a group level the magnitude of the impact of the night confinement policy on hospital patients was small. This chimes well with a previous evaluation (Braham et al, 2013) completed at a different high secure unit and strengthens their conclusion that confining high risk patients in en-suite rooms at night can contribute towards providing an efficient and effective secure mental health service.

An externally imposed structure to the night-time period was expected to change patient sleep patterns but it was not clear a priori whether sleep hygiene would improve or deteriorate. The results showed that sleep hygiene improve for some, and worsened for others, but improvement was a little more common than deterioration. The post-confinement data collection period took place around 12 weeks after confinement began but it may be that this was too soon for patients to settle into a new sleep pattern/routine. When NC was implemented, many patients had been in the same sleep pattern for many years and the disruption to this pattern caused by NC may have taken longer than 12 weeks to settle down. Anecdotal evidence gathered after 5 months of the NC policy in operation suggested that some patients who had initially found it very difficult to adjust to a new sleep pattern were now sleeping better than they ever had. In sum, sleep hygiene did not deteriorate in a consistent manner across the group.

Broadly speaking, the ward climate in the hospital compared favourably against the norms for high secure in England. Scores on all three subscales were at least as high as the norms from Rampton Hospital. In addition, ward climate was not affected in any consistent manner by NC and there were no significant changes in any of the subscales between the two time points.

Patients were broadly positive about their experiences of NC when they were asked about it after NC began. The post-NC responses on the attitudes survey were largely positive and were in sharp contrast to the pre-NC responses from the same respondents. It is clear that before NC commenced patients were apprehensive about the impact it would have and were pessimistic about the outcome of such a substantial change to the night time period. Within three months, patients were reassured and it had become clear that their fears of a deterioration were not realised and in fact, some issues had changed for the better.

Initially, ward staff were more positive about the impact of NC on patients than were the patients themselves before NC was implemented, and their attitudes shifted to be even more positive afterwards. However, some puzzling issues remain. After NC began, staff uniformly disagreed with the view that patient's sleep had improved but this was not supported either by patients' views on the matter or the PSQI data from patients. Whilst sleep for some patients had not improved (and had in fact deteriorated for some), sleep had improved for many patients and so the staff view may have presented a somewhat biased impression of patients' sleep quality. It is likely that the staff responses reflected their knowledge of one or two patients whose sleep hygiene had suffered rather than an objective assessment of all patients on their ward.

Of greater concern however, was the finding that, despite being largely positive about the impact of NC on patients, the staff view of the NC policy itself was unequivocally negative. Every staff respondent, without exception, expressed a negative view overall both before and after confinement was implemented. The fact that there was no change at all in attitudes from pre- to post-NC (when responses on all other items changed at least a little) implies that the staff response to this item was irrespective of the impact of NC when it was implemented and was driven more by concerns about the policy that were independent of the impact on patients. On the one hand, staff attitudes concerning the policy are of relatively lesser concern compared to that of the patients; nevertheless, front line staff are responsible for implementing the policy on wards and if ward staff are fundamentally opposed to the policy, this may emerge in the way the policy is implemented, may impact staff-patient relationships and fuel feelings of conflict between staff and the organisation. 
From an organisational standpoint, asking a workforce to do something that they are manifestly opposed to doing has been known to create problems. Nevertheless, it is still unclear as to why staff attitudes towards the NC policy is overwhelmingly negative. Anecdotal evidence indicated that staff objections to the NC policy were more ideologically and conceptually driven; more specifically, staff may not have been comfortable with the idea that a hospital could lock patients in their rooms, and particularly when the policy was driven by financial concerns. In spite of agreeing that patients benefitted from NC in many ways, the idea of NC still did not sit well with ward staff and there is a clear need to better understand this issue from the point of view of ward staff.

The study was subject to some limitations. With a varied patient population and clear differences between wards, a broad evaluation such as this is always more effective and more informative when carried out on the entire hospital rather than on a selection of wards. However, owing to a number of methodological and procedural issues, it was not possible to collect data from all wards. As a result, the eight wards in the hospital that first started to implement NC were not included in the evaluation, including all six of the high dependency and admissions wards. Not only did this result in a slightly skewed sample but it also restricted the number of patients who could be approached to participate and ultimately only 30 patients competed the study. The repeated measures design of the study is powerful and maximised the effectiveness of the limited data that was available; nevertheless, a relatively small sample of participating patients makes an interpretation of the results less solid. However, taken together with findings from a similar study at a different high secure service (Braham et al., 2013), the findings converge on the view that the impact of a night confinement policy on patients is minimal and may contribute to the provision an effective high secure mental health service.

\section{Conclusion}

Night confinement has been a controversial policy in high secure mental health services in England and its implementation was driven by financial concerns rather than motives based on patient care. As a result, there was a strong need to evaluate the impact of such a policy on patients. Our study suggests that the impact of the night confinement policy on patients has been minimal. The broad spectrum of measures that were utilised in this project included aspects of attitudes, sleep, and behaviour, and there were no negative effects of confinement shown on any of the indices. Indeed, some indices provided evidence of benefits of the imposition of night confinement. Nevertheless, it is important that the hospital continues to focus effort on understanding the implications of the policy from the point of view of ward staff. Importantly, patients' attitudes towards the issue of confinement at night is very broadly positive.

\section{Authors}

Dr Simon Chu is a Research Fellow and Kimberley McNeill is a Research Associate, both are based at Ashworth Research Centre, Ashworth Hospital, Mersey Care NHS Trust, Maghull, UK and School of Psychology, University of Central Lancashire, Preston, UK. Dr Karen M. Wright is a Principal Lecturer, based at School of Health, University of Central Lancashire, Preston, UK. Anthony Hague is a Modern Matron, based at Ashworth Hospital, Mersey Care NHS Trust, Maghull, UK. Dr Tracy Wilkins is a HSS Research Lead, based at Ashworth Research Centre, Ashworth Hospital, Mersey Care NHS Trust, Maghull, UK. 


\section{References}

Beazley, P., \& Gudjonsson, G. (2011). Motivating inpatients to engage with treatment: The role of depression and ward atmosphere. Nordic Journal of Psychiatry, 65(2), 95-100.

Blackburn, R., \& Renwick, S. J. (1996). Rating scales for measuring the interpersonal circle in forensic psychiatric patients. Psychological Assessment, 8(1), 76-84.

Braham, L.G., Heasley, J.F., \& Akiens, S. (2013). An evaluation of night confinement in a high secure hospital. Mental Health Review Journal, 18 (1), $21-31$.

Buysse, D. J., Reynolds, C. F., Monk, T. H., Berman, S. R., \& Kupfer, D. J. (1989). The Pittsburgh Sleep Quality Index - a new instrument for psychiatric practice and research. Psychiatry Research, 28(2), 193-213.

Hall, M., Meaden, A., Smith, J. \& Jones, C. (2001). Brief report: The development and psychometric properties of an observer-rated measure of engagement with mental health services. Journal of Mental Health, 10, 457-465.

HMSO (1992). Report of the Committee of Inquiry into Complaints about Ashworth Hospital, 1. London: HMSO.

Howells, K., Tonkin, M., Milburn, C., Lewis, J, Draycot, S., Cordwell, J., Price, M., Davies, S., \& Schalast, N. (2009). The EssenCES measure of social climate: A preliminary validation and normative data in UK high secure hospital settings. Criminal Behaviour and Mental Health, 19, 308-320

Krause, M.S. (1967). Behavioral indexes of motivation for treatment. Journal of Counselling Psychology, 14, 426-435.

McCoy, J. G., \& Strecker, R. E. (2011). The cognitive cost of sleep lost. Neurobiology of Learning and Memory, 96(4), 564-582.

Schalast N, Redies M, Collins M, Stacey J, Howells K (2008) EssenCES, a short questionnaire for assessing the social climate of forensic psychiatric wards. Criminal Behaviour and Mental Health, 18(1), 49-58.

Seibel, C.A., \& Dowd, E.T. (1999). Reactance and therapeutic noncompliance. Cognitive Therapy and Research, 23, 373-379.

Tetley, A., Jinks, M., Huband, N., \& Howells, K. (2011). A Systematic Review of Measures of Therapeutic Engagement in Psychosocial and Psychological Treatment, Journal of Clinical Psychology, 67(9), 927941.

Tonkin, M., Howells, K., Ferguson, E., Clark, A., Newberry, M., \& Schalast, N. (2012). Lost in Translation? Psychometric properties and construct validity of the English Essen Climate Evaluation Schema (EssenCES) social climate questionnaire. Psychological Assessment, 24(3), 573-580. 